\title{
A GENERIC RESULT IN VECTOR OPTIMIZATION
}

\author{
ALEXANDER J. ZASLAVSKI \\ Received 17 November 2005; Revised 19 March 2006; Accepted 24 March 2006
}

We study a class of vector minimization problems on a complete metric space such that all its bounded closed subsets are compact. We show that for most (in the sense of Baire category) problems in the class the sets of minimal values are infinite.

Copyright (c) 2006 Alexander J. Zaslavski. This is an open access article distributed under the Creative Commons Attribution License, which permits unrestricted use, distribution, and reproduction in any medium, provided the original work is properly cited.

\section{Introduction}

The study of vector optimization problems has recently been a rapidly growing area of research. See, for example, [1-5] and the references mentioned therein. In this paper, we study a class of vector minimization problems on a complete metric space such that all its bounded closed subsets are compact. This class of problems is associated with a complete metric space of continuous vector functions $\mathscr{A}$ defined below. For each $F$ from $\mathscr{A}$, we denote by $v(F)$ the set of all minimal elements of the image $F(X)=\{F(x): x \in X\}$. In this paper, we will study the sets $v(F)$ with $F \in \mathscr{A}$. It is clear that for a minimization problem with only one criterion the set of minimal values is a singleton. In the present paper, we will show that for most $F \in \mathscr{A}$ (in the sense of Baire category) the sets $v(F)$ are infinite. Such approach is often used in many situations when a certain property is studied for the whole space rather than for a single element of the space. See, for example, $[7,8]$ and the references mentioned there. Our results show that in general the sets $v(F), F \in \mathscr{A}$, are rather complicated. Note that in our paper as in many other works on optimization theory [1-6] inequalities are of great use.

In this paper, we use the convention that $\infty / \infty=1$ and denote by $\operatorname{Card}(E)$ the cardinality of the set $E$.

Let $\mathbb{R}$ be the set of real numbers and let $n$ be a natural number. Consider the finitedimensional space $\mathbb{R}^{n}$ with the Chebyshev norm

$$
\|x\|=\left\|\left(x_{1}, \ldots, x_{n}\right)\right\|=\max \left\{\left|x_{i}\right|: i=1, \ldots, n\right\}, \quad x=\left(x_{1}, \ldots, x_{n}\right) \in R^{n} .
$$


Let $\left\{e_{1}, \ldots, e_{n}\right\}$ be the standard basis in $R^{n}$ :

$$
e_{1}=(1,0, \ldots, 0), \ldots, e_{n}=(0, \ldots, 0,1) .
$$

Let $x=\left(x_{1}, \ldots, x_{n}\right), y=\left(y_{1}, \ldots, y_{n}\right) \in R^{n}$. We equip the space $R^{n}$ with the natural order and say that

$$
\begin{gathered}
x \geq y \quad \text { if } x_{i} \geq y_{i} \forall i \in\{1, \ldots, n\}, \\
x>y \quad \text { if } x \geq y, x \neq y, \\
x \gg y \quad \text { if } x_{i}>y_{i} \forall i \in\{1, \ldots, n\} .
\end{gathered}
$$

We say that $x \ll y$ (resp., $x<y, x \leq y)$ if $y \gg x$ (resp., $y>x, y \geq x$ ).

Let $(X, \rho)$ be a complete metric space such that each of its bounded closed subsets is compact. Fix $\theta \in X$.

Denote by $\mathscr{A}$ the set of all continuous mappings $F=\left(f_{1}, \ldots, f_{n}\right): X \rightarrow R^{n}$ such that for all $i \in\{1, \ldots, n\}$,

$$
\lim _{\rho(x, \theta) \rightarrow \infty} f_{i}(x)=\infty
$$

For each $F=\left(f_{1}, \ldots, f_{n}\right), G=\left(g_{1}, \ldots, g_{n}\right) \in \mathscr{A}$, set

$$
\begin{gathered}
\tilde{d}(F, G)=\sup \left\{\left|f_{i}(x)-g_{i}(x)\right|: x \in X, i=1, \ldots, n\right\}, \\
d(F, G)=\tilde{d}(F, G)(1+\tilde{d}(F, G))^{-1} .
\end{gathered}
$$

Clearly, the metric space $(\mathscr{A}, d)$ is complete.

Note that $\tilde{d}(F, G)=\sup \{\|F(x)-G(x)\|: x \in X\}$ for all $F, G \in \mathscr{A}$.

Let $A \subset R^{n}$ be a nonempty set. An element $x \in A$ is called a minimal element of $A$ if there is no $y \in A$ for which $y<x$.

Let $F \in \mathscr{A}$. A point $x \in X$ is called a point of minimum of $F$ if $F(x)$ is a minimal element of $F(X)$. If $x \in X$ is a point of minimum of $F$, then $F(x)$ is called a minimal value of $F$. Denote by $M(F)$ the set of all points of minimum of $F$ and put $v(F)=F(M(F))$.

The following proposition will be proved in Section 2.

Proposition 1.1. Let $F=\left(f_{1}, \ldots, f_{n}\right) \in \mathscr{A}$. Then $M(F)$ is a nonempty bounded subset of $(X, \rho)$ and for each $z \in F(X)$ there is $y \in v(F)$ such that $y \leq z$.

In the sequel we assume that $n \geq 2$ and that the space $(X, \rho)$ has no isolated points.

The following theorem is our main result. It will be proved in Section 4 .

THEOREM 1.2. There exists a set $\mathscr{F} \subset \mathcal{A}$ which is a countable intersection of open everywhere dense subsets of $\mathscr{A}$ such that for each $F \in \mathscr{F}$ the set $v(F)$ is infinite.

It is clear that if $X$ is a finite-dimensional Euclidean space, then $X$ is a complete metric space such that all its bounded closed subsets are compact and Theorem 1.2 holds. It is also clear that Theorem 1.2 holds if $X$ is a convex compact subset of a Banach space or if $X$ is a convex closed cone generated by a convex compact subset of a Banach space which does not contain zero. 


\section{Proof of Proposition 1.1}

Let $z \in F(X)$. We show that there is $y \in v(F)$ such that $y \leq z$. Set

$$
\Omega_{0}=\{h \in F(X): h \leq z\} .
$$

We consider the set $\Omega_{0}$ with the natural order and show that $\Omega_{0}$ has a minimal element by using Zorn's lemma.

Assume that $D$ is a nonempty subset of $\Omega_{0}$ such that for each $h_{1}, h_{2} \in D$ either $h_{1} \geq h_{2}$ or $h_{1} \leq h_{2}$. Since all bounded closed subsets of $X$ are compact, it follows from (1.4) that the set $F(X)$ is bounded from below. Together with (2.1) this implies that the set $D$ is bounded. For each integer $i \in\{1, \ldots, n\}$, set

$$
\bar{h}_{i}=\inf \left\{\lambda \in \mathbb{R}: \text { there is } x=\left(x_{1}, \ldots, x_{n}\right) \in D \text { for which } x_{i}=\lambda\right\}
$$

and set

$$
\bar{h}=\left(\bar{h}_{1}, \ldots, \bar{h}_{n}\right)
$$

Clearly, the vector $\bar{h}$ is well defined.

Let $p$ be a natural number. By (2.2) and (2.3) for each natural number $j \in\{1, \ldots, n\}$ there exists

$$
z^{(p, j)}=\left(z_{1}^{(p, j)}, \ldots, z_{n}^{(p, j)}\right) \in D
$$

such that

$$
\bar{h}_{j} \geq z_{j}^{(p, j)}-\frac{1}{p}
$$

It is clear that there is

$$
z^{(p)} \in\left\{z^{(p, j)}: j=1, \ldots, n\right\}
$$

such that

$$
z^{(p)} \leq z^{(p, j)} \quad \forall j=1, \ldots, n
$$

It follows from (2.5), (2.7), (2.2), (2.6), and (2.4) that for each $j=1, \ldots, n$,

$$
\bar{h}_{j} \leq z_{j}^{(p)} \leq \bar{h}_{j}+\frac{1}{p} .
$$

By (2.6), (2.4), and (2.1) for each integer $p \geq 1$, there is $x_{p} \in X$ such that

$$
F\left(x_{p}\right)=z^{(p)} .
$$

If the sequence $\left\{x_{p}\right\}_{p=1}^{\infty}$ is unbounded, then in view of (2.9) and (1.4) the sequence $\left\{z^{(p)}\right\}_{p=1}^{\infty}$ is also unbounded and this contradicts (2.8). Therefore the sequence $\left\{x_{p}\right\}_{p=1}^{\infty}$ 


\section{A generic result}

is bounded. Since any bounded closed set in $(X, \rho)$ is compact, there is a subsequence $\left\{x_{p_{i}}\right\}_{i=1}^{\infty}$ of the sequence $\left\{x_{p}\right\}_{p=1}^{\infty}$ which converges to some point $\bar{x} \in X$. In view of (2.8) and (2.9)

$$
F(\bar{x})=\lim _{i \rightarrow \infty} F\left(x_{p_{i}}\right)=\lim _{i \rightarrow \infty} z^{\left(p_{i}\right)}=\bar{h}
$$

and $\bar{h} \in F(X)$. Together with (2.1) and (2.2) this implies that $\bar{h} \in \Omega_{0}$. Definition (2.2) implies that $\bar{h} \leq h$ for all $h \in D$. By Zorn's lemma there is a minimal element $y \in F(X)$ such that $y \leq z$. This completes the proof of Proposition 1.1.

\section{Auxiliary results}

Proposition 3.1. Let $F=\left(f_{1}, \ldots, f_{n}\right) \in \mathscr{A}$ and let $\operatorname{Card}(v(F))=p$, where $p$ is a natural number. Then there is a neighborhood $W$ of $F$ in $(\mathscr{A}, d)$ such that $\operatorname{Card}(v(G)) \geq p$ for each $G=\left(g_{1}, \ldots, g_{n}\right) \in W$.

Proof. Let

$$
\begin{gathered}
y_{1}, \ldots, y_{p} \in v(F), \\
y_{i} \neq y_{j} \text { for each }(i, j) \in \Omega:=\{1, \ldots, p\} \times\{1, \ldots, p\} \backslash\{(i, i): i=1, \ldots, p\} .
\end{gathered}
$$

For each $i \in\{1, \ldots, p\}$, there is $x_{i} \in X$ such that

$$
F\left(x_{i}\right)=y_{i}
$$

By (3.2) and (3.3) for each $(i, j) \in \Omega$, there is $p(i, j) \in\{1, \ldots, n\}$ such that

$$
f_{p(i, j)}\left(x_{i}\right)>f_{p(i, j)}\left(x_{j}\right) .
$$

Choose $\epsilon>0$ such that

$$
f_{p(i, j)}\left(x_{i}\right)>f_{p(i, j)}\left(x_{j}\right)+4 \epsilon
$$

for all $(i, j) \in \Omega$. Set

$$
W=\{G \in \mathscr{A}: \tilde{d}(G, F) \leq \epsilon\}
$$

Let

$$
G=\left(g_{1}, \ldots, g_{n}\right) \in W .
$$

For each $i \in\{1, \ldots, p\}$, we have $G\left(x_{i}\right) \in G(X)$ and it follows from Proposition 1.1 that there is

$$
\bar{y}_{i} \in v(G)
$$

such that

$$
\bar{y}_{i} \leq G\left(x_{i}\right)
$$


Let $i \in\{1, \ldots, p\}$. By (3.8) there is $\bar{x}_{i} \in X$ such that

$$
G\left(\bar{x}_{i}\right)=\bar{y}_{i}
$$

In view of (3.7) and (3.6)

$$
\left\|G\left(\bar{x}_{i}\right)-F\left(\bar{x}_{i}\right)\right\| \leq \epsilon .
$$

It follows from (3.1), (3.2), the equality $\operatorname{Card}(v(F))=p$, and Proposition 1.1 that there is $k(i) \in\{1, \ldots, p\}$ such that

$$
F\left(\bar{x}_{i}\right) \geq y_{k(i)}
$$

By (3.3), (3.12), (3.11), (3.10), and (3.9)

$$
\begin{aligned}
F\left(x_{k(i)}\right) & =y_{k(i)} \leq F\left(\bar{x}_{i}\right) \leq G\left(\bar{x}_{i}\right)+\epsilon(1,1, \ldots, 1) \\
& \leq G\left(x_{i}\right)+\epsilon(1,1, \ldots, 1) \leq F\left(x_{i}\right)+2 \epsilon(1,1, \ldots, 1) .
\end{aligned}
$$

Together with (3.5) this implies that $k(i)=i$. Combined with (3.13), (3.10), and (3.3) this equality implies that

$$
y_{i} \leq \bar{y}_{i}+\epsilon(1,1, \ldots, 1) \leq y_{i}+2 \epsilon(1,1, \ldots, 1)
$$

It follows from this inequality, (3.5), and (3.3) that

$$
\bar{y}_{i} \neq \bar{y}_{j} \quad \text { if } i, j \in\{1, \ldots, p\} \text { satisfy } i \neq j .
$$

This completes the proof of Proposition 3.1.

Proposition 3.2. Assume that $F=\left(f_{1}, \ldots, f_{n}\right) \in \mathscr{A}, p$ is a natural number, $\operatorname{Card}(v(F))=$ $p$ and that

$$
\begin{gathered}
v(F)=\left\{y_{1}, \ldots, y_{p}\right\}, \quad x_{i} \in X, \quad F\left(x_{i}\right)=y_{i}, \quad i=1, \ldots, p, \\
y_{i} \neq y_{j} \quad \forall i, j \in\{1, \ldots, p\} \text { satisfying } i \neq j .
\end{gathered}
$$

Then for each $i=1, \ldots, p$ the inequality $F\left(x_{i}\right) \leq F(x)$ holds for all $x$ belonging to a neighborhood of $x_{i}$.

Proof. It is sufficient to consider the case with $i=1$. Clearly, for each $j \in\{2, \ldots, n\}$, there is $s(j) \in\{1, \ldots, n\}$ such that $f_{s(j)}\left(x_{1}\right)<f_{s(j)}\left(x_{j}\right)$. Choose $\epsilon>0$ such that

$$
f_{s(j)}\left(x_{1}\right)<f_{s(j)}\left(x_{j}\right)-2 \epsilon \quad \forall j \in\{2, \ldots, n\} .
$$

There is $\delta>0$ such that for each $x \in X$ satisfying $\rho\left(x, x_{1}\right) \leq \delta$ we have

$$
\left\|F(x)-F\left(x_{1}\right)\right\| \leq \frac{\epsilon}{2} .
$$


Let $x \in X$ satisfy $\rho\left(x, x_{1}\right) \leq \delta$. Then (3.18) is true. By Proposition 1.1 there exists $y \in v(F)$ such that

$$
y \leq F(x)
$$

In order to complete the proof it is sufficient to show that $y=F\left(x_{1}\right)$.

Let us assume the converse. Then there is $j \in\{2, \ldots, n\}$ such that $y=y_{j}=F\left(x_{j}\right)$. By this relation, (3.18), and (3.19)

$$
\begin{gathered}
F\left(x_{j}\right)=y_{j}=y \leq F(x) \leq F\left(x_{1}\right)+\left(\frac{\epsilon}{2}\right)(1,1, \ldots, 1), \\
f_{s(j)}\left(x_{j}\right) \leq f_{s(j)}\left(x_{1}\right)+\frac{\epsilon}{2} .
\end{gathered}
$$

This contradicts (3.17). The contradiction we have reached proves Proposition 3.2.

Proposition 3.3. Assume that $F=\left(f_{1}, \ldots, f_{n}\right) \in \mathscr{A}, \epsilon>0, p$ is a natural number and that

$$
\begin{array}{cl}
\operatorname{Card}(v(F))=p, \quad & x_{1}, \ldots, x_{p} \in X, \quad y_{i}=F\left(x_{i}\right), \quad i=1, \ldots, p, \\
& v(F)=\left\{y_{i}: i=1, \ldots, p\right\} .
\end{array}
$$

Then there exists $G=\left(g_{1}, \ldots, g_{n}\right) \in \mathscr{A}$ such that

$$
\begin{gathered}
f_{i}(x) \leq g_{i}(x), \quad x \in X, i=1, \ldots, n, \quad g_{i}\left(x_{j}\right)=f_{i}\left(x_{j}\right), \quad i=1, \ldots, n, j=1, \ldots, p, \\
\tilde{d}(F, G) \leq \epsilon \\
v(G)=\left\{G\left(x_{j}\right): j=1, \ldots, p\right\}
\end{gathered}
$$

and that for each $x \in X \backslash\left\{x_{1}, \ldots, x_{p}\right\}$ there is $j \in\{1, \ldots, p\}$ for which

$$
G(x) \geq G\left(x_{j}\right)+\epsilon \min \left\{1, \rho\left(x, x_{i}\right): i=1, \ldots, p\right\}(1,1, \ldots, 1) .
$$

Proof. For each $x \in X$ and $i=1, \ldots, n$, set

$$
g_{i}(x)=f_{i}(x)+\epsilon \min \left\{1, \rho\left(x, x_{j}\right): j=1, \ldots, p\right\}
$$

and set $G=\left(g_{1}, \ldots, g_{n}\right)$. Clearly, $G \in \mathscr{A}$,

$$
\begin{gathered}
g_{i}(x) \geq f_{i}(x), \quad x \in X, i=1, \ldots, n, \\
g_{i}\left(x_{j}\right)=f_{i}\left(x_{j}\right) \quad \text { for each } i \in\{1, \ldots, n\} \text { and each } j \in\{1, \ldots, p\}
\end{gathered}
$$

and $\tilde{d}(F, G) \leq \epsilon$. Therefore (3.22) and (3.23) hold.

Let $j \in\{1, \ldots, p\}$. We will show that $G\left(x_{j}\right) \in v(G)$. Assume that $x \in X$ and

$$
G(x) \leq G\left(x_{j}\right) .
$$


By (3.22), (3.26), and (3.28)

$$
F(x) \leq F(x)+\epsilon \min \left\{1, \rho\left(x, x_{i}\right): i=1, \ldots, p\right\}(1,1, \ldots, 1)=G(x) \leq G\left(x_{j}\right)=F\left(x_{j}\right) .
$$

Together with (3.21) this relation implies that

$$
F(x)=F\left(x_{j}\right), \quad x \in\left\{x_{i}: i=1, \ldots, p\right\}, x=x_{j} .
$$

Thus

$$
\left\{G\left(x_{j}\right): j=1, \ldots, p\right\} \subset v(G) .
$$

Assume that

$$
x \in X \backslash\left\{x_{1}, \ldots, x_{p}\right\} .
$$

By Proposition 1.1 and (3.21) there is $j \in\{1, \ldots, p\}$ such that

$$
F\left(x_{j}\right) \leq F(x) \text {. }
$$

Relations (3.22), (3.33), (3.26), and (3.32) imply that

$$
G\left(x_{j}\right)=F\left(x_{j}\right) \leq F(x)<F(x)+\epsilon \min \left\{1, \rho\left(x, x_{i}\right): i=1, \ldots, p\right\}(1, \ldots, 1) \leq G(x) .
$$

This relation implies that

$$
G(x)>G\left(x_{j}\right)+\epsilon \min \left\{1, \rho\left(x, x_{i}\right): i=1, \ldots, p\right\}(1,1, \ldots, 1)
$$

and $G(x) \notin v(G)$. Together with (3.31) this relation implies (3.24). This completes the proof of Proposition 3.3.

Proposition 3.4. Assume that $F=\left(f_{1}, \ldots, f_{n}\right) \in \mathscr{A}, p$ is a natural number,

$$
\operatorname{Card}(v(F))=p
$$

and that $\epsilon>0$. Then there exists $G \in \mathscr{A}$ such that $\tilde{d}(F, G) \leq \epsilon$ and $\operatorname{Card}(v(G))=p+1$.

Proof. Let

$$
v(F)=\left\{y_{1}, \ldots, y_{p}\right\}
$$

where $y_{1}, \ldots, y_{p} \in R^{n}$. Clearly,

$$
y_{i} \neq y_{j} \quad \text { for each } i, j \in\{1, \ldots, p\} \text { such that } i \neq j \text {. }
$$

For each $i \in\{1, \ldots, p\}$, there is $x_{i} \in X$ such that

$$
F\left(x_{i}\right)=y_{i}
$$


8 A generic result

By Proposition 3.3 there exists $F^{(1)}=\left(f_{1}^{(1)}, \ldots, f_{1}^{(n)}\right) \in \mathscr{A}$ such that

$$
\begin{gathered}
f_{i}^{(1)}(x) \geq f_{i}(x) \quad \forall x \in X, i=1, \ldots, n, \\
f_{i}^{(1)}\left(x_{j}\right)=f_{i}\left(x_{j}\right), \quad i=1, \ldots, n, j=1, \ldots, p, \\
\tilde{d}\left(F, F^{(1)}\right) \leq \frac{\epsilon}{4} \\
v\left(F^{(1)}\right)=\left\{F^{(1)}\left(x_{j}\right): j=1, \ldots, p\right\}
\end{gathered}
$$

and that for each $x \in X \backslash\left\{x_{1}, \ldots, x_{p}\right\}$ there is $j \in\{1, \ldots, p\}$ such that

$$
F^{(1)}(x) \geq F^{(1)}\left(x_{j}\right)+\epsilon \min \left\{1, \rho\left(x, x_{i}\right): i=1, \ldots, p\right\}(1,1, \ldots, 1) .
$$

It is clear that there exists a positive number

$$
\epsilon_{0}<\min \frac{\{1, \epsilon\}}{8}
$$

and that for each $i, j \in\{1, \ldots, p\}$ satisfying $i \neq j$ there exists $s(i, j) \in\{1, \ldots, n\}$ such that

$$
f_{s(i, j)}^{(1)}\left(x_{i}\right)<f_{s(i, j)}^{(1)}\left(x_{j}\right)-8 \epsilon_{0} .
$$

Choose $\delta_{0} \in(0,1 / 8)$ such that

$$
\rho\left(x_{i}, x_{j}\right) \geq 8 \delta_{0} \quad \text { for each } i, j \in\{1, \ldots, p\} \text { satisfying } i \neq j .
$$

There is $\delta_{1} \in\left(0, \delta_{0} / 2\right)$ such that for each $x \in X$ satisfying $\rho\left(x_{1}, x\right) \leq 2 \delta_{1}$

$$
\left\|F^{(1)}\left(x_{1}\right)-F^{(1)}(x)\right\| \leq \frac{\epsilon_{0}}{8} .
$$

Put

$$
\epsilon_{1}=\frac{\epsilon_{0}}{4}
$$

There is $x_{0} \in X$ such that

$$
0<\rho\left(x_{0}, x_{1}\right)<\delta_{1}
$$

By (3.50) and (3.47)

$$
x_{0} \notin\left\{x_{i}: i=1, \ldots, p\right\} .
$$

Choose a positive number

$$
\epsilon_{2}<\min \left\{\frac{\epsilon_{0} \rho\left(x_{0}, x_{1}\right)}{4}, \epsilon_{1}\right\} .
$$


Choose a positive number $\delta_{2}$ such that

$$
4 \delta_{2}<\rho\left(x_{0}, x_{1}\right)
$$

$\left|f_{i}^{(1)}\left(x_{0}\right)-f_{i}^{(1)}(x)\right| \leq \frac{\epsilon_{2}}{4} \quad$ for each $i \in\{1, \ldots, n\}$ and each $x \in X$ satisfying $\rho\left(x, x_{0}\right) \leq 4 \delta_{2}$.

Choose a positive number $\lambda$ such that

$$
\lambda \delta_{2}>2 \epsilon_{1}+2 \epsilon_{2}
$$

Set

$$
g_{1}(x)=f_{1}^{(1)}(x) \quad \text { for each } x \in X \text { satisfying } \rho\left(x, x_{0}\right)>2 \delta_{2},
$$

$g_{1}(x)=\min \left\{f_{1}^{(1)}(x), f_{1}^{(1)}\left(x_{0}\right)-\epsilon_{1}+\lambda \rho\left(x, x_{0}\right)\right\} \quad$ for each $x \in X$ satisfying $\rho\left(x, x_{0}\right) \leq 2 \delta_{2}$.

For $i \in\{2, \ldots, n\}$, set

$$
g_{i}(x)=f_{i}^{(1)}(x) \quad \text { for each } x \in X \text { satisfying } \rho\left(x, x_{0}\right)>2 \delta_{2},
$$

$g_{i}(x)=\min \left\{f_{i}^{(1)}(x), f_{i}^{(1)}\left(x_{0}\right)-\epsilon_{2}+\lambda \rho\left(x, x_{0}\right)\right\} \quad$ for each $x \in X$ satisfying $\rho\left(x, x_{0}\right) \leq 2 \delta_{2}$.

Set $G=\left(g_{1}, \ldots, g_{n}\right)$. By (3.54) and (3.55) for each $i \in\{1, \ldots, n\}$ and each $x \in X$ satisfying $\delta_{2} \leq \rho\left(x, x_{0}\right) \leq 2 \delta_{2}$,

$$
\begin{aligned}
f_{i}^{(1)}\left(x_{0}\right)-\epsilon_{1}+\lambda \rho\left(x, x_{0}\right) & \geq f_{i}^{1}\left(x_{0}\right)-\epsilon_{1}+\lambda \delta_{2} \\
& \geq f_{i}^{1}\left(x_{0}\right)+\epsilon_{1}+2 \epsilon_{2} \geq f_{i}^{(1)}(x)-\epsilon_{2} / 4+\epsilon_{1}+2 \epsilon_{2} .
\end{aligned}
$$

In view of (3.60), (3.57), and (3.59) for each $i \in\{1, \ldots, n\}$ and each $x \in X$ satisfying $\delta_{2} \leq$ $\rho\left(x, x_{0}\right) \leq 2 \delta_{2}$,

$$
g_{i}(x)=f_{i}^{(1)}(x)
$$

Together with (3.56)-(3.59) this implies that $G$ is continuous. By (3.56) and (3.58) $G \in \mathscr{A}$. Relations (3.61), (3.56), and (3.58) imply that for each $x \in X$ satisfying $\rho\left(x_{0}, x\right) \geq \delta_{2}$ we have

$$
F^{(1)}(x)=G(x)
$$

By (3.57) and (3.59) for each $x \in X$ satisfying $\rho\left(x_{0}, x\right) \leq 2 \delta_{2}$, we have

$$
G(x) \leq F^{1}(x) \text {. }
$$

Let $x \in X$ satisfy

$$
\rho\left(x, x_{0}\right) \leq \delta_{2} .
$$


By (3.56)-(3.59), (3.52), (3.64), and (3.54) for each $i \in\{1, \ldots, n\}$,

$$
\begin{aligned}
f_{i}^{(1)}(x) & \geq g_{i}(x) \geq \min \left\{f_{i}^{(1)}(x), f_{i}^{(1)}\left(x_{0}\right)-\epsilon_{1}\right\} \\
& \geq \min \left\{f_{i}^{(1)}(x), f_{i}^{(1)}(x)-\frac{\epsilon_{2}}{4}-\epsilon_{1}\right\} \geq f_{i}^{(1)}(x)-\left(\frac{5}{4}\right) \epsilon_{1}, \\
F^{(1)}(x) & \geq G(x) \geq F^{(1)}(x)-\left(\frac{\epsilon}{2}\right)(1,1, \ldots, 1) .
\end{aligned}
$$

Together with (3.62) this inequality implies that $\tilde{d}\left(F^{(1)}, G\right) \leq \epsilon / 2$. Combined with (3.42) this implies that

$$
\tilde{d}(F, G) \leq \tilde{d}\left(F, F^{(1)}\right)+\tilde{d}\left(F^{(1)}, G\right)<\frac{\epsilon}{4}+\frac{\epsilon}{2} .
$$

Let $x \in X$. We show that there exists $j \in\{0, \ldots, p\}$ such that $G(x) \geq G\left(x_{j}\right)$. There are two cases:

$$
\begin{aligned}
& \rho\left(x, x_{0}\right) \geq \delta_{2}, \\
& \rho\left(x, x_{0}\right)<\delta_{2} .
\end{aligned}
$$

Assume that (3.67) holds. Then by (3.62) $G(x)=F^{(1)}(x)$. In view of Proposition 1.1 and (3.43) there is $j \in\{1, \ldots, p\}$ such that

$$
F^{(1)}\left(x_{j}\right) \leq F^{(1)}(x)=G(x) .
$$

If $j=1$, then (3.53) implies that

$$
\rho\left(x_{j}, x_{0}\right)=\rho\left(x_{0}, x_{1}\right)>4 \delta_{2} .
$$

If $j \neq 1$, then by (3.47) and (3.50)

$$
\rho\left(x_{j}, x_{0}\right) \geq \rho\left(x_{j}, x_{1}\right)-\rho\left(x_{1}, x_{0}\right) \geq 8 \delta_{0}-\delta_{1} \geq 7 \delta_{0}>4 \delta_{2} .
$$

Thus in both cases $\rho\left(x_{j}, x_{0}\right)>4 \delta_{2}$. In view of this inequality and (3.62),

$$
F^{(1)}\left(x_{j}\right)=G\left(x_{j}\right)
$$

Together with (3.69) this equality implies that $G\left(x_{j}\right) \leq G(x)$. Assume that (3.68) holds. We will show that $G\left(x_{0}\right) \leq G(x)$. Relations (3.57) and (3.59) imply that

$$
G\left(x_{0}\right)=\left(f_{1}^{(1)}\left(x_{0}\right)-\epsilon_{1}, f_{2}^{(1)}\left(x_{0}\right)-\epsilon_{2}, \ldots, f_{n}^{(1)}\left(x_{0}\right)-\epsilon_{2}\right)=F^{(1)}\left(x_{0}\right)-\left(\epsilon_{1}, \epsilon_{2}, \ldots, \epsilon_{2}\right) .
$$

By (3.68)

$$
F^{(1)}(x) \geq F^{(1)}\left(x_{0}\right)-\left(\frac{\epsilon_{2}}{4}\right)(1,1, \ldots, 1) .
$$


By (3.68), (3.57), (3.59), (3.74), and (3.52)

$$
g_{1}(x) \geq \min \left\{f_{1}^{(1)}\left(x_{0}\right)-\frac{\epsilon_{2}}{4}, f_{1}^{(1)}\left(x_{0}\right)-\epsilon_{1}\right\}=f_{1}^{(1)}\left(x_{0}\right)-\epsilon_{1}
$$

and for $i \in\{1, \ldots, p\} \backslash\{1\}$,

$$
g_{i}(x) \geq \min \left\{f_{i}^{(1)}\left(x_{0}\right)-\frac{\epsilon_{2}}{4}, f_{i}^{(1)}\left(x_{0}\right)-\epsilon_{2}\right\}=f_{i}^{(1)}\left(x_{0}\right)-\epsilon_{2} .
$$

Together with (3.73) these inequalities imply that $G(x) \geq G\left(x_{0}\right)$. Thus we have shown that for each $x \in X$ there is $j \in\{0, \ldots, p\}$ such that $G(x) \geq G\left(x_{j}\right)$.

Now assume that $j_{1}, j_{2} \in\{0, \ldots, p\}$ satisfy

$$
G\left(x_{j_{1}}\right) \leq G\left(x_{j_{2}}\right) .
$$

We will show that $j_{1}=j_{2}$. In view of (3.47) and (3.50) for each $i \in\{2, \ldots, p\}$,

$$
\rho\left(x_{i}, x_{0}\right) \geq \rho\left(x_{i}, x_{1}\right)-\rho\left(x_{0}, x_{1}\right) \geq 8 \delta_{0}-\delta_{1}>7 \delta_{0}>4 \delta_{2} .
$$

By $(3.53) \rho\left(x_{0}, x_{1}\right)>4 \delta_{2}$. Therefore, for each $i \in\{1, \ldots, p\}$,

$$
\rho\left(x_{i}, x_{0}\right)>4 \delta_{2} \text {. }
$$

Together with (3.56) and (3.58)

$$
G\left(x_{i}\right)=F^{(1)}\left(x_{i}\right), \quad i=1, \ldots, p .
$$

If $j_{1}, j_{2} \in\{1, \ldots, p\}$, then in view of (3.77), (3.80), and (3.46) $F^{(1)}\left(x_{j_{1}}\right)=F^{(1)}\left(x_{j_{2}}\right)$ and $j_{1}=j_{2}$. Therefore we may consider only the case with $0 \in\left\{j_{1}, j_{2}\right\}$. Let $i \in\{1, \ldots, p\} \backslash\{1\}$. By (3.80)

$$
G\left(x_{i}\right)=F^{(1)}\left(x_{i}\right)
$$

By (3.46)

$$
f_{s(i, 1)}^{(1)}\left(x_{i}\right)<f_{s(i, 1)}^{(1)}\left(x_{1}\right)-8 \epsilon_{0}, \quad f_{s(i, 1)}^{(1)}\left(x_{1}\right)<f_{s(1, i)}^{(1)}\left(x_{i}\right)-8 \epsilon_{0} .
$$

It follows from (3.81), (3.82), (3.48), (3.50), (3.49), (3.57), and (3.59) that

$$
\begin{aligned}
g_{s(i, 1)}\left(x_{i}\right) & =f_{s(i, 1)}^{(1)}\left(x_{i}\right)<f_{s(i, 1)}^{(1)}\left(x_{1}\right)-8 \epsilon_{0} \\
& \leq f_{s(i, 1)}^{(1)}\left(x_{0}\right)+\frac{\epsilon_{0}}{4}-8 \epsilon_{0}<f_{s(i, 1)}^{(1)}\left(x_{0}\right)-2 \epsilon_{1} \leq g_{s(i, 1)}\left(x_{0}\right)-\epsilon_{1}, \\
g_{s(i, 1)}\left(x_{i}\right) & =f_{s(i, 1)}^{(1)}\left(x_{i}\right)>f_{s(1, i)}^{(1)}\left(x_{1}\right)+8 \epsilon_{0} \geq f_{s(i, 1)}^{(1)}\left(x_{0}\right)-\frac{\epsilon_{0}}{4}+8 \epsilon_{0}>g_{s(1, i)}\left(x_{0}\right)+7 \epsilon_{0} .
\end{aligned}
$$

These inequalities imply that the inequality $G\left(x_{i}\right) \leq G\left(x_{0}\right)$ does not hold and that the inequality $G\left(x_{0}\right) \leq G\left(x_{i}\right)$ does not hold too. Together with (3.77) and the inclusion $0 \in$ $\left\{j_{1}, j_{2}\right\}$ this implies that

$$
\left\{j_{1}, j_{2}\right\} \subset\{0,1\}
$$


By (3.80)

$$
G\left(x_{1}\right)=F^{(1)}\left(x_{1}\right), \quad g_{s}\left(x_{1}\right)=f_{s}^{1}\left(x_{1}\right), \quad s=1, \ldots, n .
$$

Relations (3.79) and (3.44) imply that there is $q \in\{1, \ldots, p\}$ such that

$$
F^{(1)}\left(x_{0}\right) \geq F^{(1)}\left(x_{q}\right)+\epsilon \min \left\{1, \rho\left(x_{0}, x_{i}\right): i=1, \ldots, p\right\}(1,1, \ldots, 1) .
$$

It follows from (3.50), (3.48), and (3.86) that

$$
F^{(1)}\left(x_{q}\right) \leq F^{(1)}\left(x_{0}\right) \leq F^{(1)}\left(x_{1}\right)+\left(\epsilon_{0} / 8\right)(1, \ldots, 1) .
$$

Together with (3.46) this implies that $q=1$. Combined with (3.86) this equality implies that

$$
F^{(1)}\left(x_{0}\right) \geq F^{(1)}\left(x_{1}\right)+\epsilon \min \left\{1, \rho\left(x_{0}, x_{i}\right): i=1, \ldots, p\right\}(1,1, \ldots, 1) .
$$

By (3.47), (3.50), (3.88), and (3.85) for $i \in\{1, \ldots, p\} \backslash\{1\}$,

$$
\begin{gathered}
\rho\left(x_{0}, x_{i}\right) \geq \rho\left(x_{1}, x_{i}\right)-\rho\left(x_{1}, x_{0}\right) \geq 8 \delta_{0}-\delta_{1} \geq 7 \delta_{0}, \\
\min \left\{1, \rho\left(x_{0}, x_{i}\right): i=1, \ldots, p\right\}=\rho\left(x_{0}, x_{1}\right), \\
F^{(1)}\left(x_{0}\right) \geq F^{(1)}\left(x_{1}\right)+\epsilon \rho\left(x_{0}, x_{1}\right)(1,1, \ldots, 1)=G^{(1)}\left(x_{1}\right)+\epsilon \rho\left(x_{0}, x_{1}\right)(1,1, \ldots, 1) .
\end{gathered}
$$

Relations (3.90), (3.59), and (3.52) imply that for $i \in\{1, \ldots, p\} \backslash\{1\}$

$$
\begin{gathered}
g_{i}^{(1)}\left(x_{1}\right)+\epsilon \rho\left(x_{0}, x_{1}\right) \leq f_{i}^{(1)}\left(x_{0}\right)=g_{i}\left(x_{0}\right)+\epsilon_{2}, \\
g_{i}^{(1)}\left(x_{1}\right) \leq g_{i}\left(x_{0}\right)+\epsilon_{2}-\epsilon \rho\left(x_{0}, x_{1}\right) \leq g_{i}\left(x_{0}\right)-\epsilon_{2} .
\end{gathered}
$$

Relations (3.90) and (3.57) imply that

$$
g_{1}\left(x_{1}\right)+\epsilon \rho\left(x_{0}, x_{1}\right) \leq f_{1}^{(1)}\left(x_{0}\right)=g_{1}\left(x_{0}\right)+\epsilon_{1} .
$$

By this relation, (3.50), (3.48), (3.49), and (3.85)

$$
g_{1}\left(x_{0}\right)=f_{1}^{(1)}\left(x_{0}\right)-\epsilon_{1} \leq f_{1}^{(1)}\left(x_{1}\right)+\frac{\epsilon_{0}}{8}-\epsilon_{1}=f_{1}^{(1)}\left(x_{1}\right)-\frac{\epsilon_{0}}{8}=g_{1}\left(x_{1}\right)-\frac{\epsilon_{0}}{8} .
$$

Then each of the inequalities $G\left(x_{0}\right) \leq G\left(x_{1}\right), G\left(x_{1}\right) \leq G\left(x_{0}\right)$ does not hold. Together with (3.84), the inclusion $0 \in\left\{j_{1}, j_{2}\right\}$ and (3.77) this implies that $j_{1}=j_{2}=0$. Thus we have shown that if $j_{1}, j_{2} \in\{0, \ldots, p\}$ and if $G\left(x_{j_{1}}\right) \leq G\left(x_{j_{2}}\right)$, then $j_{1}=j_{2}$. Therefore $\operatorname{Card}(v(G))=$ $p+1$. Proposition 3.4 is proved.

\section{Proof of Theorem 1.2}

Lemma 4.1. Let $F \in \mathscr{A}, p \geq 1$ be an integer and let $\epsilon>0$. Then there exists an open nonempty set

$$
u \subset\{H \in \mathscr{A}: \tilde{d}(F, H) \leq \epsilon\}
$$

such that for each $G \in \mathcal{U}$ the inequality $\operatorname{Card}(v(G)) \geq p+1$ holds. 
Proof. If for each $G \in \mathscr{A}$ satisfying $\tilde{d}(F, G)<\epsilon$ we have $\operatorname{Card}(v(G)) \geq p+1$, then put

$$
\boldsymbol{u}=\{H \in \mathscr{A}: \tilde{d}(F, H)<\epsilon\} .
$$

Assume that there is $G_{0} \in \mathscr{A}$ such that

$$
\tilde{d}\left(F, G_{0}\right)<\epsilon, \quad \operatorname{Card}\left(v\left(G_{0}\right)\right) \leq p .
$$

By Proposition 3.4 there exists $G_{1} \in \mathscr{A}$ such that

$$
\tilde{d}\left(F, G_{1}\right)<\epsilon, \quad \operatorname{Card}\left(v\left(G_{1}\right)\right)=p+1 .
$$

By Proposition 3.1 there exists an open neighborhood $U$ of $G_{1}$ in $\mathscr{A}$ such that

$$
u \subset\{H \in \mathscr{A}: \tilde{d}(H, G)<\epsilon\}
$$

and that for each $G \in \mathcal{U}$ the inequality $\operatorname{Card}(v(G)) \geq p+1$ holds. Lemma 4.1 is proved.

Proof of Theorem 1.2. Let $p$ be a natural number. By Lemma 4.1 for each $F \in \mathscr{A}$ and each integer $i \geq 1$ there exists an open nonempty set

$$
\mathcal{u}(F, i, p) \subset\left\{H \in \mathscr{A}: \tilde{d}(F, H) \leq(2 i)^{-1}\right\}
$$

such that for each $H \in \mathcal{U}(F, i, p)$ the inequality $\operatorname{Card}(v(H)) \geq p$ holds. Define

$$
\mathscr{F}=\cap_{p=1}^{\infty} \bigcup\{U(F, i, p): F \in \mathscr{A}, i \geq 1 \text { is an integer }\} .
$$

It is clear that $\mathscr{F}$ is a countable intersection of open everywhere dense subsets of $\mathscr{A}$ and that for each $G \in \mathscr{F}$ the set $v(G)$ is infinite. Theorem 1.2 is proved.

\section{References}

[1] G.-Y. Chen, X. Huang, and X. Yang, Vector Optimization, Lecture Notes in Economics and Mathematical Systems, vol. 541, Springer, Berlin, 2005.

[2] J. P. Dauer and R. J. Gallagher, Positive proper efficient points and related cone results in vector optimization theory, SIAM Journal on Control and Optimization 28 (1990), no. 1, 158-172.

[3] M. Ehrgott and X. Gandibleux (eds.), Multiple Criteria Optimization: State of the Art Annotated Bibliographic Surveys, International Series in Operations Research \& Management Science, vol. 52, Kluwer Academic, Massachusetts, 2002.

[4] J. Jahn, Vector Optimization. Theory, Applications, and Extensions, Springer, Berlin, 2004.

[5] T. Tanino, Stability and sensitivity analysis in convex vector optimization, SIAM Journal on Control and Optimization 26 (1988), no. 3, 521-536.

[6] Z. Wei, L. Qi, and J. R. Birge, A new method for nonsmooth convex optimization, Journal of Inequalities and Applications 2 (1998), no. 2, 157-179. 


\section{A generic result}

[7] A. J. Zaslavski, Generic existence of solutions of nonconvex optimal control problems, Abstract and Applied Analysis 2005 (2005), no. 4, 375-421.

[8] _ Turnpike Properties in the Calculus of Variations and Optimal Control, Nonconvex Optimization and Its Applications, vol. 80, Springer, New York, 2006.

Alexander J. Zaslavski: Department of Mathematics, The Technion-Israel Institute of Technology, 32000 Haifa, Israel

E-mail address: ajzasl@tx.technion.ac.il 